\section{Responses to steroids and bronchodilators in COPD in the ISOLDE trial: the fat lady sings on}

\section{N J Gross}

Two new findings using data from the ISOLDE trial are presented in this issue of Thorax: (1) patients with COPD cannot be separated into discrete corticosteroid responders and non-responders, and (2) the response of an individual patient with COPD to a bronchodilator challenge on a single occasion does not predict whether or not the patient will benefit subsequently from that agent. Consistency is needed between North America and Europe as to the diagnosis of COPD and the criteria for inclusion in COPD trials.

$\mathrm{T}$ he main results of the ISOLDE trial have been published ${ }^{1}$ and are now part of the canon of knowledge about COPD—inhaled corticosteroids (ICS) do not change the rate of decline of $\mathrm{FEV}_{1}$ in COPD. This finding is consistent with that of other similar large long term trials, ${ }^{2-4}$ so the question has been settled. As in some of the other studies, there may be decreases in the frequency and severity of acute exacerbations, and quality of life may be modestly improved. These questions are being addressed in separate ongoing long term trials.

Despite the rule that the primary and secondary outcomes of a trial must be prestated and set in stone in the protocol, trials such as ISOLDE are so large and so carefully planned, executed, and monitored that the huge amounts of data they generate offer many opportunities to examine questions other than the prestated outcomes-questions that are scientifically important but which are unlikely ever to find funding as primary outcomes. In the absence of any methodological aspect of the trial that would invalidate it from being used to answer a different question, it is appropriate to mine the data for any and all other useful information they may provide. Sometimes disparaged as "data dredging", the meticulous review of the data for other insights seems rather to be an economical use of a precious resource-namely, good data which should be exploited for all they are worth. Two important papers in this issue of Thorax illustrate this.

The paper by Burge et al bears on one of the most important remaining issues concerning the long term use of ICS in COPD—is it possible to identify "steroid responders" by a short preliminary course of oral prednisolone? The issue is important because a very large proportion of patients with COPD are already receiving long term ICS, often without a clear rationale or a solid experimental basis; this is a matter of some concern.

Inhaled corticosteroids are probably not innocuous, particularly in the relatively large dosages that are sometimes being used in COPD, ${ }^{6}$ and particularly when one considers that ICS may be used continuously for 10 or even 20 years in the COPD population who are older and in whom the common side effects of steroid therapy such as osteoporosis and muscular atrophy are already common. Potential adverse effects of ICS such as these might take many years of continuous use to become evident, and none of the published studies of ICS has exceeded 40 months, which is not nearly long enough to be confident of the safety of their long term use. By the widespread and indiscriminate use of ICS in COPD, might we be making a Faustian compact in which modest short term benefits in some patients are being traded for what may be an epidemic of serious adverse effects over the long term? This possibility, admittedly portrayed in its most disastrous form, makes it most important to determine whether there is an identifiable subgroup of patients with COPD who are not responsive to corticosteroid therapy. If so, these patients should perhaps be saved from even the potential risk of long term ICS use. Previous attempts to identify steroid responder and non-responder groups have been largely unsuccessful, as cited by Burge et al. ${ }^{5}$

The unique design of the ISOLDE study, which included a 2 week trial of oral prednisolone before randomisation into the main 3 year trial, made it possible to address this question with good statistical power. In the present report the authors found no statistical association between the change in $\mathrm{FEV}_{1}$ resulting from the course of oral prednisolone and any outcome over the subsequent 3 years of treatment with ICS or placebo. This finding invalidates the traditional recommendation that one should perform a steroid trial before instituting ICS on a long term basis. It could be argued that the increase in $\mathrm{FEV}_{1}$ during the short prednisolone trial might not be an appropriate way to identify responder and non-responder groups. Indeed, the $\mathrm{FEV}_{1}$ does not seem to be a measurement that correlates well with clinically relevant outcomes. ${ }^{7}$ However, it is hard to know what other outcome(s) of a short trial of prednisolone would be better than the $\mathrm{FEV}_{1}$, or even practical in the clinic. If the purpose of a preliminary prednisolone trial is to guide the clinician in his/her decision whether or not to administer ICS to the patient with COPD, the indicator of its success or failure must be one that clinicians can readily apply in their daily practice. This rules out the measurement of markers of COPD-type inflammation in airway secretions, cells, or breath condensates that might conceivably predict steroid nonresponsiveness. ${ }^{8}$ One day, perhaps, but such assays are neither validated nor realistic in clinical practice at present.

The present trial ${ }^{5}$ and all previous evidence seems to suggest, as Burge and colleagues state, that "patients with COPD cannot be separated into . . corticosteroid responders and nonresponders". Whether this is because there really are no subgroups of steroid responders and non-responders, or whether there are but we have not yet discovered how to identify and differentiate them, is uncertain. I believe the right course is to keep an open mind about the possibility that there may be responder and non-responder subgroups and to continue to seek ways to identify and characterise them, if they exist. Meanwhile, I regretfully agree with the conclusion that corticosteroid trials are not diagnostically helpful in primary care.

The paper by Calverley and colleagues in this issue of Thorax, 9 also based on data from the ISOLDE trial, addresses the important question of whether poor bronchodilator responsiveness is a valid criterion for the diagnosis of COPD or predicts disease progression. Although the ISOLDE population was selective due to the inclusionary criterion of a poor bronchodilator response, Calverley et al show that "classifying patients as [bronchodilator] 'responders' and 'nonresponders' can be misleading and does not predict disease outcome". 
The problem surrounding the notion of non-responsiveness to bronchodilators in COPD is the legacy of a document generated by a group of academic lung specialists who met in 1958 to establish diagnostic criteria for chronic obstructive lung diseases. They defined what we now call COPD as "a group of diseases with persistent or irreversible obstructive lung disease ${ }^{\prime \prime} .{ }^{10}$ However, it has been known for as long as spirometry has been routinely performed that this definition is flawed ${ }^{11}{ }^{12}$ and that patients with clinical features that every practising respiratory physician would call COPD are often capable of a "significant" bronchodilator response. The only thing one can confidently say about the bronchodilator responsiveness of patients with COPD is that it is not, on average, as great as that in patients with asthma. But the overlap between the two diagnoses in this respect is so great that they cannot be reliably distinguished on this basis. ${ }^{13}{ }^{14}$ Bronchodilator responses of patients with COPD can, in fact, be quite large with some current agents, ${ }^{15}$ nor might they be elicited by a bronchodilator challenge on a single occasion as shown by Calverley et al and previously by others. ${ }^{16-18}$ If one still doubts that a bronchodilator response is typical in patients who meet the clinical criteria of COPD, one should look at fig 2 in the paper by Calverley and colleagues ${ }^{9}$ where what looks as though it might have been a perfect Gaussian distribution of bronchodilator responsiveness is sharply truncated on the right hand side by the prior exclusion of potential subjects who had a response to salbutamol of more than $10 \%$ of predicted $\mathrm{FEV}_{1}$. Despite this, the mean $\mathrm{FEV}_{1}$ response following salbutamol was $4-6 \%$ predicted, or about $170 \mathrm{ml}$, which is both statistically and clinically significant and was even greater when salbutamol and ipratropium were used together.

The implication which Calverley and colleagues make here, and which is made in another recent paper in almost identical words, ${ }^{19}$ is that the response of an individual COPD patient to a bronchodilator challenge on a single occasion does not predict whether the patient will or will not benefit from that agent subsequently. The size of the response in patients with COPD is not only inconsistent over time but, whatever limit one sets on it, is arbitrarywhether $12 \%$ of baseline $e^{20}$ or $10 \%$ of predicted. ${ }^{1}$ Perhaps equally important, they argue that a bronchodilator response should not be used as an exclusionary criterion in clinical trials involving patients who otherwise conform to the diagnostic criteria of COPD. I strongly agree. Doing so not only perpetuates a 45 year old misconception, but it irrationally excludes a subset of patients with COPD who happened to be tested on a day when they responded rather better than they may have responded on another day.

There is a divergence of views between regulatory agencies in Europe and North America on this matter which hurts both of us. In North America a limited response to bronchodilators is no longer required as an inclusionary criterion of COPD trials. Of course, as a North American, I say let us compromise-do it our way! But it would be best for practitioners on both sides of the Atlantic if we could be consistent in how we diagnose and admit patients to COPD trials. The paper by Calverley and colleagues suggests rather strongly that COPD trials should include all patients who meet the clinical criteria of COPD that we can all agree upon and should not exclude patients on the mistaken notion of bronchodilator non-responsiveness that goes back to an era when spirometric tests were very rarely performed.

Two papers, two important messages. Does the fat lady have another encore for us?

Thorax 2003;58:647-648

\section{Author's affiliation}

N J Gross, Hines VA Hospital, Stritch-Loyola School of Medicine, Chicago, IL 60141, USA; Nicholas.gross@med.va.gov

\section{REFERENCES}

1 Burge PS, Calverley PMA, Jones PW, et al. Randomised, double-blind, placebo controlled study of fluticasone propionate in patients with moderate to severe chronic obstructive pulmonary disease, the ISOLDE trial. BM 2000;320: 1297-303

2 Pauwels RA, Lofdahl CG, Laitinen LA, et al. Long-term treatment with inhaled budesonide in persons with mild chronic obstructive pulmonary disease who continue smoking. $N$ Engl J Med 1999;340: 1948-53.
3 Vestbo J, Sorensen $P$, Lange $P$, et al. Long-term effect of inhaled budesonide in mild and moderate chronic obstructive pulmonary disease. Lancet 1999;353:1819-23.

4 Lung Health Study Research Group. Effect of inhaled triamcinolone on the decline in pulmonary function in chronic obstructive pulmonary disease. N Engl J Med 2000;343: 1902-9.

5 Burge PS, Calverley PMA, Jones PW, et al. Prednisolone response in patients with chronic obstructive pulmonary disease: results from the ISOLDE study. Thorax 2003;58:654-8.

6 Calverley PMA, Pauwels R, Vestbo J, et al. Combined salmeterol and fluticasone in the treatment of chronic obstructive pulmonary disease, a randomized controlled trial. Lancet 2003;361:449-56.

7 Jones PW, Quirk FH, Baveystock CM, et al. A self-complete measure of health status for chronic airflow limitation, the St George's Respiratory Questionnaire. Am Rev Respir Dis 1992;145:1321-7.

8 Barnes PJ. Inhaled steroids are not beneficial in chronic obstructive pulmonary disease. Am J Respir Crit Care Med 2000;161:342-4.

9 Calverley PMA, Burge PS, Spencer A, et al. Bronchodilator reversibility testing in chronic obstructive pulmonary disease. Thorax 2003;58:659-64.

10 Ciba Foundation Guest Symposium. Terminology, definitions and classification of chronic pulmonary emphysema and related conditions. Thorax 1959;44:286-99.

11 Curtis JK, Liska AP, Rasmussen HK, et al. The bronchospastic component in patients with chronic bronchitis and emphysema. JAMA 1966;197:693-6.

12 Moron JW, Turnbull KW. The reversibility of chronic bronchitis, asthma, and emphysema. Dis Chest 1968;53:126-32.

13 Brand PL, Quanjer PH, Postma DS, et al. Interpretation of bronchodilator response in patients with obstructive airways disease, the Dutch Chronic Non-Specific Lung Disease (CNSLD) Study Group. Thorax 1992;47:429-36.

14 Dompeling E, Van Schayck CP, Molema J, et al. A comparison of six different ways of expressing the bronchodilating response in asthma and COPD, reproducibility and dependence on pre-bronchodilator $\mathrm{FEV}_{1}$. Eur Respir J 1992;5:975-81.

15 Casaburi R, Mahler DA, Jones PW, et al. A long-term evaluation of once-daily inhaled tiotropium in chronic obstructive pulmonary disease. Eur Respir J 2002;19:217-24.

16 Anthonisen NR, Wright EC. Bronchodilator response in chronic obstructive pulmonary disease. Am Rev Respir Dis 1986; 133:814-9.

17 Gross NJ, Co E, Skorodin MS. Cholinergic bronchomotor tone in COPD, estimates of its amount in comparison with that in normal subjects. Chest 1989:96:984-7.

18 Nisar M, Earis JE, Pearson MG, et al. Acute bronchodilator trials in chronic obstructive pulmonary disease. Am Rev Respir Dis 1992;146:555-9.

19 Tashkin D, Kesten S. Long-term benefits with tiotropium in COPD patients with and without acute bronchodilator responses. Chest 2003; 123:1441-51

20 American Thoracic Society. Standards for the diagnosis and care of patients with chronic obstructive pulmonary disease. Am J Respir Crit Care Med 1995;152:S77-120. 


\section{Breathing exercises and asthma}

\section{Thomas}

\section{Evidence to support the efficacy of complementary and alternative medicines in asthma is limited. A study of the effect of two breathing exercises (Butekyo and pranayama) in patients with asthma reported in this issue of Thorax contributes to the evidence base, but further controlled studies are needed.}

$\mathrm{T}$ here is considerable lay and professional interest in nonpharmacological treatments for asthma, with reports that up to one third of people with asthma resort to complementary and alternative medicines (CAM). ${ }^{1}$ The evidence-based review undertaken for the British guidelines on the management of asthma ${ }^{2}$ found the current evidence for the presence or absence of efficacy of many CAM interventions to be inadequate, and further controlled studies are encouraged. Breathing exercises and yoga have been widely used to treat asthma in Eastern and Western societies for many years, and generally centre on manipulating the respiratory pattern to reduce respiratory frequency and hyperventilation. The Butekyo breathing technique, based on the barely tenable scientific premise that asthma is caused by hyperventilation, makes sweeping claims for effectiveness in asthma. ${ }^{3}$ In spite of anecdotal reports of benefit given wide coverage in the lay press, the limited scientific scrutiny currently afforded to this technique has indicated more modest improvements in asthma outcomes, with two small controlled studies showing some benefits in symptoms and bronchodilator use although little effect on other measures of asthma severity. A Cochrane review of breathing exercises for asthma ${ }^{4}$ (updated in 2000 and currently undergoing revision) found it was not possible to draw reliable conclusions on the effectiveness of breathing retraining from current published evidence. Since this review, there have been reports in this journal of limited beneficial effects in symptoms and airways hyperresponsiveness to methacholine resulting from yoga breathing exercises, ${ }^{5}$ and of improvements in asthma related quality of life resulting from a community physiotherapy based breathing retraining programme in a subgroup with symptoms suggestive of dysfunctional breathing. ${ }^{6} \mathrm{It}$ is still, however, far from clear whether or not breathing exercises can improve asthma outcomes, in which groups they may be effective, or what the mechanism of effect may be. In this issue of Thorax Cooper et $\mathrm{al}^{7}$ report a further controlled trial investigating the effectiveness of Butekyo and a device mimicking pranayama yoga exercises, and conclude that the Butekyo method can improve symptoms and reduce bronchodilator use but not by affecting lung function or bronchial hyperresponsiveness.

There is clearly a need for controlled studies on representative patient groups in this area. This study recruited symptomatic adult patients treated with inhaled corticosteroids who had reversible airflow obstruction and hyperresponsiveness to inhaled methacholine. The patients were recruited from an asthma volunteer database and by advertisement, so it is not certain that the study group is necessarily representative of the wider asthma population. The investigators have made efforts to address the real methodological problems that exist in controlling and blinding multifaceted CAM treatments. The study attempted to control for non-specific intervention effects independent of breathing pattern alterations by comparing a variety of asthma outcomes in the Butekyo group with those in the groups using an active and inactive "placebo" pranayama breathing training device. The Butekyo group did, however, receive a higher level of professional contact than the other groups, so it is possible that the effects of professional attention may act as a confounding influence on the improved outcomes reported. Statistically significant improvements in symptom scores were reported for the Butekyo group, although the magnitude and clinical relevance of the improvement was less clear with the unvalidated scoring tool used. Reductions in bronchodilator use were seen in the Butekyo group, although since the Butekyo training process strongly discourages patients from using bronchodilators, the reduction may represent a learning effect and may not be appropriate as a surrogate marker of asthma control in this situation. No differences were seen in other objective outcome measures-including bronchial hyperresponsiveness, lung function, and asthma exacerbations-nor were there any differences in the ability to reduce inhaled corticosteroid dosage, although the study may not have been powered adequately to show this.

If subjective benefits are indeed found in relation to Butekyo and other types of breathing retraining, it is necessary to attempt clarification of the mechanisms of improvement. International consensus has defined asthma as an inflammatory condition characterised by airways hyperresponsiveness and variable airflow limitation, ${ }^{8}$ and subjective improvements need to be related to objective measures of asthma severity. Asthma is a complex disease and the relationship between objective physiological measures and the patients' subjective experience of their condition is far from simple. It has, for instance, been shown that psychosocial and emotional factors influence asthma symptoms and asthma related health status independently of asthma severity, ${ }^{9}$ and that the relationship between airflow obstruction and symptoms is very weak $^{10}$ with some patients experiencing high levels of symptoms in spite of normal or near normal lung function. The question of whether improvements in asthma symptoms associated with breathing retraining may result from indirect effects on emotional or psychological factors cannot be answered from the current evidence. It has been reported that up to $30 \%$ of adults with asthma in the community, of all severity levels, may have symptoms suggestive of functional breathing disorders, ${ }^{11}$ raising the possibility that symptom improvements following breathing retraining interventions may relate to treatment of a co-existent functional problem rather than of asthma per se. This possibility is strengthened by the results of this study, in which subjective symptom improvements are not matched by changes in objective parameters of airways calibre or hyperresponsiveness. Other studies have, however, shown improvements in bronchial hyperresponsiveness in relation to breathing exercises. ${ }^{5}$ No currently published studies have investigated the effects of breathing retraining on parameters of airway inflammation. Preliminary evidence from an animal model suggests that repeated dry air hyperventilation can result in airways inflammation and hyperreactivity, ${ }^{13}$ so raising a possible link between abnormal breathing and asthma. It is also unclear whether different types of breathing retraining interventions-such as physiotherapist based programmes for treating hyperventilation, different schools of yoga, and the Butekyo method-have similar effects and act by similar mechanisms. 
In spite of the various highly effective inhaled and oral pharmacological options available for the treatment of asthma, the goals of asthma management are not currently being met, with high levels of potentially avoidable morbidity revealed in surveys. ${ }^{14}$ For a variety of incompletely understood reasons, many of our patients are unable or unwilling to comply with our recommended treatment, and many wish to explore non-pharmacological treatment avenues such as breathing exercises. In contrast to the wealth of high quality evidence informing pharmacological decision making in asthma, often driven by the pharmaceutical industry, there is a paucity of information for rational decision making in non-pharmacological treatments. Although progress is being made in improving this evidence base, large holes still exist in our knowledge and understanding and further controlled studies are required to confirm effectiveness and clarify mechanisms of benefit if found.

Thorax 2003:58:649-650

\section{Author's affiliation}

M Thomas, GPIAG Research Fellow, Department of General Practice, University of Aberdeen; Primary Care Advisor,

Gloucestershire Research and Development Support Unit; GP, Minchinhampton, Gloucestershire; Hospital Practitioner, Respiratory Medicine, Stroud Hospital, Gloucestershire, UK

Correspondence to: $\operatorname{Dr} M$ Thomas, Cotswold Cottage, Oakridge, Stroud, Gloucestershire GL67NZ, UK; mikethomas@doctors.org.uk

\section{REFERENCES}

1 Ernst E. Complementary therapies for asthma: what patients use. J Asthma 1998:35:667-71.

2 British Thoracic Society/Scottish Intercollegiate Guideline Network. British guidelines on the management of asthma. Thorax 2003;58(Suppl I): i 1-94.

3 Stalmatski A. Freedom from asthma: Butekyo's revolutionary treatment. London: Kyle Cathie Ltd, 1997.

4 Holloway E, Ram FSF. Breathing exercises for asthma (Cochrane review). The Cochrane Library. Issue 3. Oxford: Update Software, 2000.

5 Manocha R, Marks GB, Kenchington P, et al. Sahaja yoga in the management of moderate to severe asthma: a randomised controlled trial. Thorax 2002;57: 110-5.
6 Thomas M, McKinley RK, Freeman E, et al. Breathing retraining for dysfunctional breathing in asthma: a randomised controlled trial. Thorax 2003;58: 1 10-5.

7 Cooper S, Oborne J, Nelson S, et al. Effect of two breathing exercises (Butekyo and pranayama) in asthma: a randomised controlled trial. Thorax 2003;58:674-9.

8 International Consensus Report on the diagnosis and treatment of asthma. Eur Respir J 1992:5:641.

9 Rimington LD, Davies DH, et al. Relationship between anxiety, depression, and morbidity in adult asthma patients. Thorax 2001:56:266-71.

10 Teeter JG, Bleecker ER. Relationship between airway obstruction and respiratory symptoms in adult asthmatics. Chest 1998;1 13:277

11 Thomas M, McKinley RK, Freeman E, et al. Prevalence of dysfunctional breathing in patients treated for asthma in primary care: a cross-sectional survey. BM 2001;322:1098-100.

12 Singh V, Wisniewski A, Britton J, et al. Effect of yoga breathing exercise (pranayama) on airways reactivity in subjects with asthma. Lancet 1990;335:1381-3.

13 Davis MS, Freed AN. Repeated hyperventilation causes peripheral airways inflammation, hyperreactivity and impaired bronchodilation in dogs. Am J Respir Crit Care Med 2001;164:785-9.

14 Rabe KF, Vermeire PA, Soriano JB, et al. Clinical management of asthma in 1999: the Asthma Insights and Reality in Europe (AIRE) study. Eur Respir J 2000;16:802-7. has been proposed as a possible source, but systematic studies of coronaviruses in a wide spectrum of wild and semidomestic species are not yet complete. RNA viruses tend to evolve rapidly and coronaviruses frequently undergo homologous recombination, so that coinfection with an established human coronavirus and SARS CoV could lead to emergence of new virus species combining various features of the parental strains.

The clinical picture of SARS CoV infection continues to emerge, but patients in the early stages may not complain of respiratory symptoms and my not be febrile; influenza-like symptoms, abdominal pain, and diarrhoea are common, ${ }^{3}$ followed by transient high fever and then by ARDS, progressive respiratory failure, spontaneous pneumomediastinum, and disseminated intravascular coagulopathy (DIC). Full recovery may take weeks or months. ${ }^{4}$ The WHO definitions were established to assist in the definition of hospital cases and have a sensitivity of only $26 \%$ in the detection of non-hospitalised patients defined by seroconversion. ${ }^{3}$ Particular problems with SARS CoV include:

- the diagnosis is difficult;

- it spreads fast in hospital wards (especially if nebulisers are used or emergency resuscitation is performed);

- even fit, healthy people (including hospital workers) are affected; and

- there are no proven specific treatments. 
SARS has knock-on effects on the care of other patients and disrupts the lives of relatives and hospital staff alike. A major SARS outbreak in the UK could effectively close down the health service.

In this issue of Thorax, Chan et al $l^{5}$ describe the clinical features of 115 patients (including five doctors and 18 nurses) with SARS admitted to a single hospital in Hong Kong, beginning in March 2003. Their mean age was 41 years, and the crude mortality was $15.7 \%$ with one third of deaths occurring more than 3 weeks after onset of symptoms. Intriguingly, Chan et al show that diabetes, cardiac disease, and age are strongly predictive of an adverse outcome, mirroring a smaller previous study of patients mainly from the Amoy Gardens housing block. In this other study $40 \%$ of those with ARDS $(n=15)$ had chronic hepatitis B infection compared with 5\% of the 60 patients who did not develop ARDS.

These observations raise intriguing questions about why some patients become very ill and die while others have mild disease and survive. Certainly, young healthy people infected with SARS CoV rarely become very ill, ${ }^{6}$ and those already elderly ${ }^{7}$ or in poor health are at increased risk when a viral or bacterial pneumonia develops. However, it is also possible that some of the patients with pre-existing disease may have heightened innate immune responses that augment the immunopathological response to SARS and thus leads to more severe pulmonary infiltration, ARDS, and death. It is also possible that the immunological traits that lead to chronic disease-for example, hepatitis B infection or immune organ damage-also adversely influence the immunopathological response to SARS CoV infection.
Experience with smallpox and polio shows that a highly effective vaccine is essential for global elimination of an infectious disease and that an animal reservoir makes elimination hard or impossible. Vaccine development is a worldwide priority, funded by US Federal support for industrial partners using three distinct approaches. A vaccine would be likely to prevent systemic spread; there are successful vaccines for some veterinary coronavirus infections and it would be possible to test vaccines in non-human primates. ${ }^{2}$ However, success is not guaranteed and anticoronavirus immunity can even increase disease severity-for example, in coronavirus induced feline peritonitis. The existing human coronavirus common cold agents are able to re-infect despite low variability, and prolonged viral shedding in SARS patients (about $70 \%$ of patients are still positive at day 21 on stool samples) despite good serological responses $(60 \%$ seroconversion by day 21 and virtually $100 \%$ by day $30^{4}$ ) indicates that a specific immune response may not be capable of terminating infection.

The SARS outbreak has important lessons for us all. Epidemics of this type do not respect national borders, have a large impact on tourism, travel and trade, and potentially have devastating effects in poor countries with insufficient infrastructure. The unprecedented speed of international and national collaboration undoubtedly contributed greatly to limiting the impact of SARS, and the WHO and respective governments must be praised for their incisive and energetic leadership. What will happen to SARS during the next 6-9 months is guesswork-a major worldwide epidemic might develop this coming winter or the outbreak could die down. Certainly, there will be more outbreaks of respiratory viral disease in the future, and we need to be well prepared for such events.

Thorax 2003;58:650-651

\section{Author's affiliation}

P J M Openshaw, Department of Respiratory Medicine, St Mary's Campus, National Heart and Lung Division, Imperial College, London, UK; p.openshaw@ic.ac.uk

\section{REFERENCES}

1 Drosten C, Gunther S, Preiser W, et al. Identification of a novel coronavirus in patients with severe acute respiratory syndrome. N Engl J Med 2003;348:196776.

2 Fouchier RA, Kuiken T, Schutten M, et al. Aetiology: Koch's postulates fulfilled for SARS virus. Nature (London) 2003;423:240.

3 Rainer TH, Cameron PA, Smit D, et al. Evaluation of WHO criteria for identifying patients with severe acute respiratory syndrome out of hospital: prospective observational study. BM 2003;326:1354-8.

4 Peiris JS, Chu CM, Cheng VC, et al. Clinical progression and viral load in a community outbreak of coronavirus-associated SARS pneumonia: a prospective study. Lancet 2003;361:1767-72.

5 Chan JWM, Ng CK, Chan YH, et al. Short term outcome and risk factors for adverse clinical outcomes in adults with severe acute respiratory syndrome (SARS). Thorax 2003;58:696-9

6 Hon KL, Leung CW, Cheng WT, et al. Clinical presentations and outcome of severe acute respiratory syndrome in children. Lancet 2003;361:1701-3.

7 Donnelly CA, Ghani AC, Leung GM, et al. Epidemiological determinants of spread of causal agent of severe acute respiratory syndrome in Hong Kong. Lancet 2003;361:1761-6.

\section{Closing the NETT on lung volume reduction surgery}

\section{P M A Calverley}

\section{The National Emphysema Treatment Trial (NETT) of lung volume reduction surgery in patients with COPD has shown that surgery can and should be evaluated on a par with other forms of treatment.}

M ost textbooks and many physicians now use the term "chronic obstructive pulmonary disease" (COPD) to define airflow obstruction that results from a variable combination of small airways disease and loss of elastic recoil due to emphysema. A detailed knowledge of the underlying pathology does not normally influence the treatment prescribed, with one important exception. ${ }^{1}$ Patients who have large space occupying bullae visible on their plain chest radiograph can experience significant improvements in lung function and exercise capacity if these lesions are resected, a treatment that is now well established. ${ }^{2}$ Initial attempts to extend this approach to include the resection of gross emphysematous areas of lungs were scorned by physiologists as being irrational and were associated with significant perioperative morbidity and mortality. ${ }^{3}$ The pressures of a lengthening lung transplantation waiting list led Cooper and colleagues to revisit this approach using modern techniques of intensive care and better surgical methods of strengthening the previously suspect suture lines between friable areas of lung. Their report of significant improvements in spirometry, breathlessness, and 6-minute walking distance after surgery compared with historical controls had a 
dramatic effect on thoracic surgical practice in the USA. ${ }^{4}$ Their findings were replicated by others using a variety of surgical approaches and techniques and were reported in a series of uncontrolled case studies $^{5}$ which suggested variable benefit when meta-analysed. ${ }^{6}$ After some debate, this procedure is now known as lung volume reduction surgery (LVRS). Detailed physiological testing before and after surgery showed that there was a significant improvement in resting lung volumes in most cases, together with less dynamic hyperinflation during exercise, improved diaphragmatic mechanics secondary to changes in chest wall configuration, ${ }^{8}$ and increased lung elastic recoil in the remaining lung. ${ }^{9}$ Theoretical models were developed to explain how lung volume reduction could improve expiratory flow, irrespective of the distribution of emphysema. ${ }^{10}$ Finally, several small randomised controlled trials confirmed the efficacy of LVRS in terms of sustained improvements in spirometry, exercise capacity, and health status. ${ }^{11-13}$

Unlike medical treatments which are strictly regulated and must demonstrate sustained benefits without unacceptable risk, surgical treatments have traditionally been introduced on the basis of sustained short term benefit and LVRS was no exception. However, despite the patchy nature of the longer term follow up data, it became clear that the improvement seen after surgery was not permanent and, in some cases, the return to baseline conditions was more rapid than anticipated from the normal decline in lung function known to occur in these patients. ${ }^{14}$ More worryingly, the rapid uptake of LVRS was accompanied by a steep increase in the reported 90 day mortality rate, rapidly reaching the alarming figures which had originally led to the procedure being discontinued. ${ }^{15}$

At this point something quite unusual but very appropriate happened. A unique coalition was formed between the NHLBI and the principal healthcare providers in the USA who introduced a moratorium on performing surgery of this kind outside the large prospective randomised controlled clinical trial, which they agreed to fund. This was the National Emphysema Treatment Trial (NETT), the results of which were reported initially as an interim analysis of high risk cases ${ }^{16}$ and which have now been reported both as an intention to treat analysis ${ }^{17}$ and in a companion paper addressing the cost effectiveness of the procedure. $^{18}$

Of the 3777 patients screened, 1218 were finally randomised, 580 eventually receiving surgery and 562 routine medical care. All patients underwent 6-10 weeks of pulmonary rehabilitation before entry to the study, performed cycle ergometry breathing 30\% oxygen and standardised pulmonary function testing, and completed the disease specific St George's Respiratory Questionnaire (SGRQ), a general health questionnaire, and a dyspnoea questionnaire. Emphysema distribution was graded by visual scoring of the high resolution CT scan as being homogeneous or heterogeneous, with or without upper lobe predominance of the disease. Physiological and symptomatic evaluations were conducted at 6 and 12 months and annually thereafter. Primary outcomes were all cause mortality and maximum exercise capacity. Given the risks inherent in the surgery, a higher than usual clinically significant change was established a priori-namely, an increase in maximum exercise capacity of 10 watts and an 8 point change in the SGRQ score. ${ }^{19}$ Adherence to treatment and to the pulmonary rehabilitation programme at home was monitored by telephone contact and in the clinic, and all patients were nonsmokers when studied.

Patient groups were well matched (mean age 66.6 years, mean $\mathrm{FEV}_{1}$ 26.8\% predicted, mean TLco $28.3 \%$ predicted) and were not hypercapnic $\left(\mathrm{PaCO}_{2}\right.$ $5.75 \mathrm{kPa}$ ). The total SGRQ score was around 53, a value lower than might be expected given the degree of airflow obstruction but compatible with successful pulmonary rehabilitation. The 90 day mortality was $7.9 \%$ in those randomised to surgery compared with $1.9 \%$ in those undergoing routine medical treatment. Improvements in exercise capacity of more than 10 watts occurred in $28 \%$ of surgically treated patients at 6 months and were still present in $15 \%$ at 2 years compared with $4 \%$ and $3 \%$, respectively, in the medically treated group. Early in the trial a high risk group of patients with homogeneous disease on the CT scan and an FEV and/or TLCo below 20\% predicted were identified as having an unacceptably high early mortality and no further patients of this type were recruited. In the remaining 1078 patients surgery was still significantly more hazardous by 90 days $(5.2 \%$ versus $1.2 \%$ mortality in the medical group) but mortality did not differ over the follow up period. Significantly greater changes in $\mathrm{FEV}_{1}$, health status, and the degree of dyspnoea were seen in the surgically treated patients, all showing an initial improvement with a later deterioration compared with a steady deterioration in these variables in those undergoing medical treatment.

When patients were stratified post hoc for the presence of upper lobe predominant disease and by their initial exercise impairment before randomisation, four subgroups emerged. Patients with upper lobe predominant emphysema and a low exercise capacity showed the greatest and best sustained improvements in all physiological and symptomatic variables and also had a significantly better survival experience than similar patients randomised to medical treatment. In contrast, those without upper lobe predominance of disease and a preserved exercise capacity faired scarcely better than the high risk group previously identified. The remaining two groups lay between these extremes with no benefit in mortality but significant improvements in the degree of health status impairment, spirometry, and exercise capacity.

The companion report ${ }^{18}$ examined the healthcare costs associated with this treatment which were substantial, amounting to $\$ 190000$ per quality adjusted life year (QALY) at 3 years and $\$ 53000$ at 10 years. Unsurprisingly, the most cost effective treatment was directed at those with upper lobe predominant disease and a low exercise capacity (\$98 000 per QALY at 3 years and $\$ 21000$ per QALY at 10 years). The 10 year data, adjusted for the likely survival in this population, extrapolated the benefits seen at 3 years and assumed that the treatment differences observed were maintained over this time-both rather imponderable issues in patients such as these. By comparison, coronary artery bypass surgery costs $\$ 64000$ per QALY gained (2002 prices).

There are many lessons to be learned from the NETT study. Firstly, important improvements in exercise capacity and health status are possible in patients with severe emphysema by reducing the operating lung volume at which these patients breathe. The changes in exercise capacity and well being can be dramatic even when the spirometric improvement is small, an important lesson which is applicable to all COPD treatments. These benefits can be achieved surgically without an unacceptable mortality risk, at least in patients in whom surgery is performed according to the NETT protocols and attention is paid to previous rehabilitation and patient selection. The distribution of disease and prior exercise capacity are important determinants of operative success. This suggests that more comprehensive imaging and exercise studies will be needed if we are to characterise COPD patients properly in future clinical trials and in our clinical practice. An impaired exercise capacity is not just a marker of poor prognosis, ${ }^{20}$ but also appears to define the patients with the most to gain from treatment of their underlying disease. However, we should be cautious about all the conclusions drawn in this study as some of the most important are based on a post hoc analysis of predictor variables, a source of concern to statisticians ${ }^{21}$ but less worrying to clinicians who are likely to be impressed by the biological plausibility of the conclusions drawn. Inclusion of a comprehensive prospective cost effectiveness 
analysis also emphasises the economic impact of advanced COPD and the need to offer surgery only to those patients in whom the benefit can be best justified, given the scarcity of healthcare resources.

Future analysis of this important dataset is likely to provide many new insights and to generate further hypotheses that will need to be tested. Perhaps most importantly of all, the conduct of the NETT study has shown that surgery can and should be evaluated on a par with other forms of treatment. Only when this is done can we be certain that our intervention as doctors helps rather than harms our patients

Thorax 2003;0:651-653

\section{Author's affiliation}

\section{REFERENCES}

1 Pauwels RA, Buist AS, Calverley PMA, et al. Global strategy for the diagnosis, management and prevention of chronic obstructive pulmonary disease. Am J Respir Crit Care Med 2001;163:1256-76.

2 Morgan MDL. Bullous lung disease. In: Calverley PMA, Pride NB, eds. Chronic obstructive pulmonary disease. London: Chapman and Hall, 1995: 547-60.

3 Brantigan OC, Muller EC. Surgical treatment of pulmonary emphysema. Am Rev Respir Dis 1959;80:194-202.

4 Cooper JD, Patterson GA, Sundaresan RS, et al. Results of 150 consecutive bilateral lung volume reduction procedures in patients with severe emphysema. J Thorac Cardiovasc Surg 1996;112:1319-29.

5 Davies L, Calverley PM. Lung volume reduction surgery in chronic obstructive pulmonary disease. Thorax 1996;51 (Suppl 2):S29-34.

6 Young J, Fry-Smith A, Hyde C. Lung volume reduction surgery (LVRS) for chronic obstructive pulmonary disease (COPD) with underlying severe emphysema. Thorax 1999;54:779-89.

7 Martinez FJ, De Oca MM, Whyte RI, et al. Lung-volume reduction improves dyspnea, dynamic hyperinflation, and respiratory muscle function. Am J Respir Crit Care Med 1997; 155: 1984-90.

8 Jubran A, Laghi F, Mazur M, et al. Partitioning of lung and chest-wall mechanics before and after lung-volume-reduction surgery. Am J Respir Crit Care Med 1998; 158:306-10.

9 Sciurba FC, Rogers RM, Keenan R, et al. Improvement in pulmonary function and elastic recoil after lung-volume reduction surgery for diffuse emphysema. N Engl J Med 2003;334:1095-9

10 Fessler HE, Permutt S. Lung volume reduction surgery and airflow limitation. Am J Respir Crit Care Med 1998;157:715-22.

11 Criner GJ, Cordova FC, Furukawa S, et al. Prospective randomized trial comparing bilateral lung volume reduction surgery to pulmonary rehabilitation in severe chronic obstructive pulmonary disease. Am J Respir Crit Care Med 1999; 160:2018-27.

12 Geddes D, Davies M, Koyama H, et al. Effect of lung-volume-reduction surgery in patients with severe emphysema. N Engl J Med 2000;343:239-45.

13 Goldstein RS, Todd TRJ, Guyatt G, et al. Influence of lung volume reduction surgery (LVRS) on health realted quality of life in patients with chronic obstructive pulmonary patients with chronic obstructive pul
disease. Thorax 2003;58:405-10.

14 Brenner M, McKenna RJ, Gelb AF, et al. Rate of $\mathrm{FEV}_{1}$ change following lung volume reduction surgery. Chest 1998;113:652-9.

15 Albert RK, Lewis S, Wood D, et al. Economic aspects of lung volume reduction surgery. aspects of lung volume reductic

16 National Emphysema Treatment Trial Research Group. Patients at high risk of death after lung-volume-reduction surgery. $N$ Engl J Med 2001;345:1075-83.

17 National Emphysema Treatment Trial Research Group. A randomized trial comparing lung-volume reduction surgery with comparing lung-volume reduction surgery $N$ medical therapy for severe emphys
Engl J Med 2003;348:2059-73.

18 National Emphysema Treatment Trial Research Group. Cost effectiveness of lung-volume reduction surgery for patients with severe emphysema. N Engl J Med 2003:348:2092-102.

19 Jones PW. Interpreting thresholds for a clinically significant change in health status in asthma and COPD. Eur Respir J 2002;19:398-404

20 Oga T, Nishimura K, Tsukino M, et al. Analysis of the factors related to mortality in chronic obstructive pulmonary disease: role of exercise capacity and health status. Am J Respir Crit Care Med 2003;167:544-9.

21 Ware JH. The National Emphysema Treatment Trial: how strong is the evidence? N Engl J Med 2003;348:2055-6.

\section{LUNG ALERT}

\section{A new test for latent tuberculosis infection?}

A Ewer K, Deeks J, Alvarez L, et al. Comparison of T-cell-based assay with tuberculin skin test for diagnosis of Mycobacterium tuberculosis infection in a school tuberculosis outbreak. Lancet 2003;361:1168-73

$\mathrm{T}$ his study compared the enzyme linked immunospot assay (ELISPOT) with tuberculin skin testing (TST) for the diagnosis of latent tuberculosis infection (LTBI) in low preva-

lence settings. The ELISPOT assay measures interferon gamma secretion by blood mononuclear cells to ESAT-6, an antigen present in Mycobacterium tuberculosis but not in $M$ bovis or environmental mycobacteria. 535 students were tested in a large tuberculosis outbreak in a UK school. Although agreement between the tests was high (89\%), ELISPOT correlated significantly more closely with $M$ tuberculosis exposure than did TST based on duration of exposure $(p=0.007)$ and measures of proximity to the single index case $(p=0.002)$. TST was significantly more likely to be positive in BCG vaccinated than in non-vaccinated students. The authors conclude that ELISPOT offers a more accurate approach than TST for the identification of patients with LTBI, and is more precise at targeting preventative treatment.

Interpretation of studies in this area is difficult because of the lack of a gold standard for diagnosing LTBI. There are no comparative studies between ELISPOT and QuantiFERON, an existing assay which measures the interferon response to PPD in whole blood. Although the TST requires a return visit for interpretation, it does not require phlebotomy, analysis within a few hours, laboratory expertise, or expensive equipment like an ELISPOT reader. Studies are required to assess the cost/benefit ratio of ELISPOT and its positive predictive value for the subsequent development of tuberculosis.

K Dheda 